\title{
Poly(diacetylene) Monolayers Studied with a Fluorescence Scanning Near-Field Optical Microscope
}

\author{
Marco H. P. Moers, ${ }^{*}, \dagger$ Hermann E. Gaub, ${ }^{\ddagger}$ and Niek F. van Hulst ${ }^{\dagger}$ \\ Department of Applied Physics, University of Twente, P.O. Box 217, \\ 7500 AE Enschede, The Netherlands, and Physics Department, Technical University of \\ Munich, 8046 Garching, Germany
}

Received February 18, 1994. In Final Form: May 25, $1994^{\otimes}$

\begin{abstract}
A novel and powerful method to study the optical properties of thin lipid films which a resolution superior to confocal microscopy is presented. With a scanning near-field optical microscope, fluorescence images of a Langmuir-Blodgett film of diethylene glycol diamine pentacosadiynoic amide are obtained with a lateral resolution of $100 \mathrm{~nm}$. Simultaneously a force image is measured that gives the topography. Therefore it is possible to correlate a high-resolution fluorescence micrograph with the topological structure of the polymer film. Polymer domains that do not fluoresce are visible in the force image, while polarization dependence, an optical property not afforded by atomic force microscopy, is clearly demonstrated with a high resolution in the near-field optical image. This combination of fluorescence scanning near field optical microscopy and force microscopy provides additional information on the structure of the polymer film that can be valuable in the research of Langmuir-Blodgett films.
\end{abstract}

\section{Introduction}

Thin lipid films are widely studied in different fields like biology, biochemistry, and material science. Among these, molecular polydiacetylene films attract attention because of their striking optical, electrical, mechanical, and chemical properties. For instance, they combine a high optical density and intense intrinsic fluorescence with a high anisotropy ${ }^{1,2}$ At the same time the cross-linking of the molecules increases the mechanical stability.

The properties of polymeric films have been studied with numerous techniques such as spectroscopy, ${ }^{3}$ microfluorescence, ${ }^{1,2,4} \mathrm{X}$-ray ${ }^{5}$ and electron ${ }^{6-8}$ diffraction, and scanning probe techniques like scanning tunneling microscopy (STM) -11 $^{-11}$ and atomic force microscopy (AFM) ${ }^{4,12-19}$ The first two techniques give information

\footnotetext{
$\dagger$ University of Twente.

₹ Technical University of Munich.
}

(1) Göbel, H. D.; Gaub, H. E.; Möhwald, H. Chem. Phys. Lett. 1987, 138,441 .

(2) Bubeck, C.; Tieke, B.; Wegner, G. Ber. Bunsen-Ges. Phys. Chem. $1982,86,495$.

(3) Warta, R.; Sixl, H. J. Chem. Phys. 1988, 88, 95.

(4) Goettgens, B. M.; Tillmann, R. W.; Radmacher, M.; Gaub, H. E. Langmuir 1992, 8, 1768 .

(5) Kjaer, K; Als-Nielsen, J;; Helm, C.; Tippman-Krayer, P.; Möhwald, H. J. Phys. Chem. 1989, 93, 3200.

(6) Fischer, A.; Sackmann, E. Nature 1985, 313, 299.

(7) Lieser, G.; Tieke, B.; Wegner, G. Thin Solid Films 1980, 68, 77.

(8) Garoff, S.; Deckman, H. W.; Dunsmuir, J. H.; Alvarez, M. S.;

Bloch, J. M. J. Phys. (Paris) 1986, 47, 701.

(9) Coombs, J. H.; Pethica, J. B; Welland, M. E. Thin Solid Films $1988,159,293$.

(10) Braun, H. G.; Fuchs, H.; Schrepp, W. Thin Solid Films 1988, 159,301 .

(11) Hörber, J. K. H.; Lang, C. A.; Hänseh, T. W.; Heckl, W. M." Möhwald, H. Chem. Phys. Lett. 1988, 145, 151.

(12) Binnig, G.; Quate, C. F.; Gerber, Ch. Phys. Rev. Lett. 1986, 56, 930.

(13) Weisenhorn, A. L.; Gaub, H. E.; Hansma, H. G.; Sinsheimer, R.

L.; Kelderman, G. L.; Hansma, P. K. Scanning Microsc. 1990, 4, 511.

(14) Weisenhorn, A. L.; Egger, M.; Ohnesorge, F.; Gould, S. A. C.;

Heyn, S.-P.; Hansma, H. G.; Sinsheimer, R. L.; Gaub, H. E.; Hansma,

P. K. Langmuir 1991, 7, 8 .

(15) Hansma, H. G.; Gould, S. A. C.; Hansma, P. K.; Gaub, H. E.;

Longo, M. L.; Zasadzinski, J. A. N. Langmuir 1991, 7, 1051.

(16) Meyer, E.; Howald, L.; Overney, R. M.; Heinzelmann, H.;

Frommer, J.; Guntherodt, H.-J.; Wagner, T.; Schier, H.; Roth, S. Nature $1991,349,398$.

(17) Putman, C. A. J.; Hansma, H. G.; Gaub, H. E.; Hansma, P. K. Langmuir 1992, 8, 3014 .

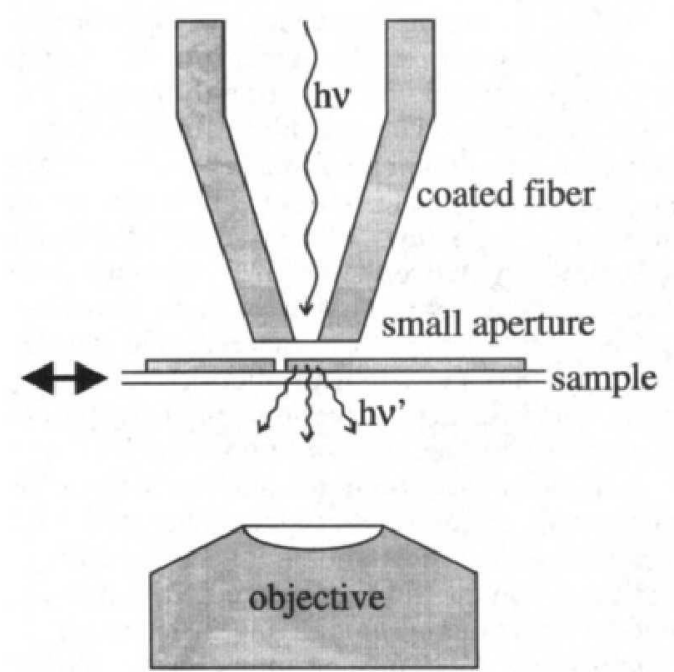

Figure 1. A schematic of the fluorescence SNOM. A small aperture, acting as a light source, is scanned close to a surface while exciting only a small volume of fluorophore at a time. The fluorescence of this small volume is detected with an inverted microscope.

about the optical properties of the films, whereas the latter give information on the structure.

During the last years a rather new technique is being developed that combines the high resolution of scanning probe microscopes with the specific contrast of conventional optical microscopy. In scanning near-field optical microscopy (SNOM) ${ }^{20-23}$ a small aperture is scanned close to a surface (i.e. in the near field) acting as a subwavelength-sized source. Consequently, the sample is illuminated only in the direct vicinity of the aperture, such that the resolution in the optical image is mainly determined by the size of the aperture and the distance between aperture and sample, and not by the wavelength, like in far-field optics.

(18) Tillmann, R. W.; Radmacher, M.; Gaub, H. E.; Kenney, P.; Ribi, H. O.J. Phys. Chem. 1993, 97, 2928.

(19) Radmacher, M.; Tillmann, R. W.; Fritz, M.; Gaub, H. E. Science $1992,257,1900$.

(20) Pohl, D. W.; Denk, W.; Lanz, M. Appl. Phys. Lett. 1984, 44, 651. (21) Harootunian, A.; Betzig, E.; Isaacson, M.; Lewis, A. Appl. Phys. Lett. 1986, 49, 674 .

(22) Betzig, E.; Trautman, J. K.; Harris, T. D.; Weiner, J. S.; Kostelak, R. L. Science 1991, 251, 1468.

(23) Betzig, E.; Trautman, J. K. Science 1992, 257, 189. 

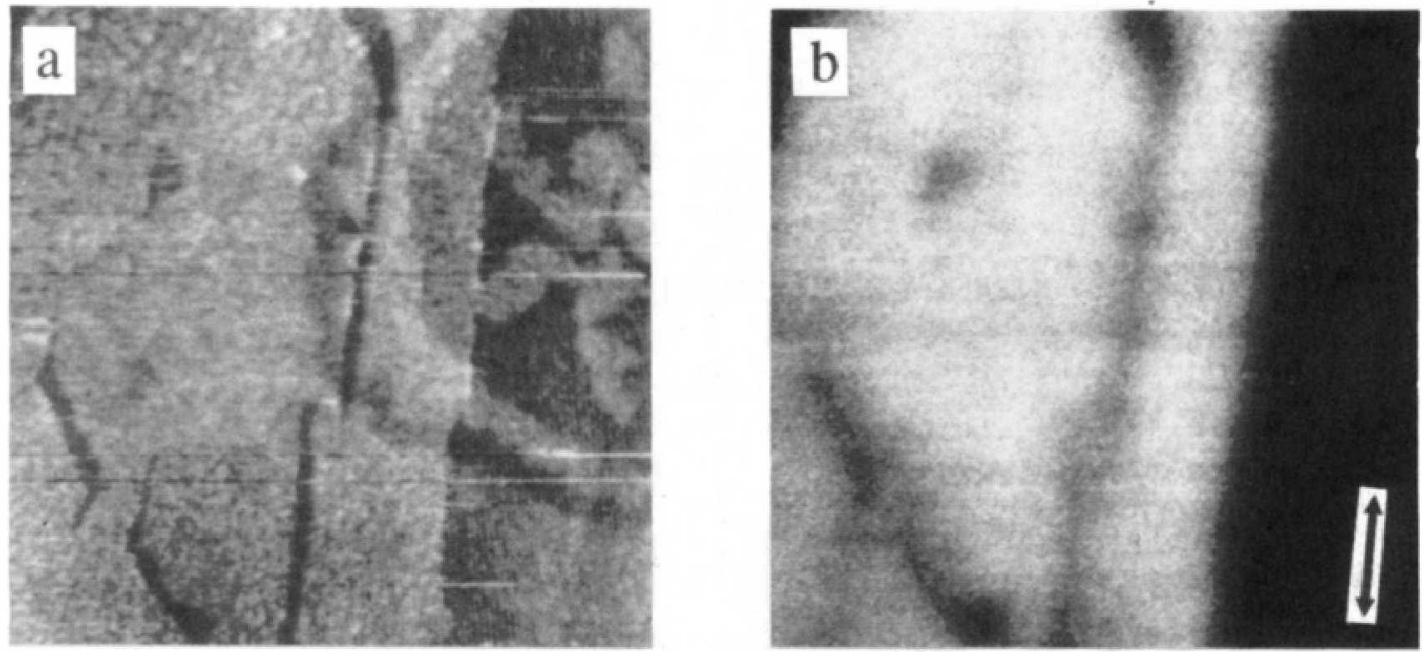

Figure 2. A $4.4 \mu \mathrm{m} \times 4.4 \mu \mathrm{m}$ scan of a LB film of DPDA showing (a) the shear force and (b) the near-field fluorescence image. Some fractions, noticeable in the topographic image (a) appear not to fluoresce. The arrow in part b denotes the incident polarization.

One of the most interesting features of such an instrument for biologists and biochemists is the operation as a fluorescence microscope. ${ }^{24,25}$ Because but a small volume of fluorophore is excited at a time, not only the optical resolution is improved but also photobleaching is reduced due to the short exposure time.

In practice, tapered optical fibers with an opaque aluminum coating on the sides are widely used as apertures with a size down to tens of nanometers. In order to operate this small and fragile light source near the surface, some distance regulation is of great importance. A feedback based on shear force ${ }^{26,27}$ has proven to be a good method to keep the probe within a few nanometers of the surface. Because this method relies on a constant distance between probe and sample, the topography of the sample is measured simultaneously with the near-field optical image. Thus the high-resolution optical image can directly be compared with the topography of the sample.

In this paper a polymerized Langmuir-Blodgett film, diethylene glycol diamine pentacosadiynoic amide (DPDA) ${ }^{18}$ is studied with a fluorescence scanning near-field optical microscope. Optical properties of the sample will be investigated with a resolution higher than that available with confocal microscopy, while force images are simultaneously recorded.

\section{Experimental Section}

Lipid Films. The sample is diethylene glycol diamine pentacosadiynoic amide (DPDA), polymerized by UV radiation. The lipid was a kind gift of Biocircuits, Sunnyvale, CA. The preparation is the same as described previously. ${ }^{18}$ The film has been transferred to a regular microscope glass slide by standard Langmuir-Blodgett techniques. The transfer speed was $20 \mu \mathrm{m} / \mathrm{s}$ vertically. The transfer ratio was close to 1 , which means that a monolayer of polymerized DPDA is transferred onto the substrate. The subphase was MilliQ water at $8^{\circ} \mathrm{C}$. The lateral pressure for polymerization was $30 \mathrm{mN} / \mathrm{m}$.

Scanning Near-Field Optical Microscope, In Figure 1a schematic of the SNOM setup is shown. A tapered, aluminumcoated optical fiber with a small aperture has been produced using standard techniques. ${ }^{22}$ Approximately $5 \mathrm{~mW}$ of the 488$\mathrm{nm}$ or 515-nm line of an argon ion laser is coupled into the untapered end of the multimode fiber, while the polarization of

(24) Betzig, E.; Chichester, R. J. Bioimaging 1993, 1, 129.

(25) Betzig, E.; Chichester, R. J. Science 1993, 262, 1422

(26) Toledo-Crow, R.; Yang, P. C.; Chen, Y.; Vaez-Iravani, M. Appl. Phys. Lett. 1992, 60, 2957.

(27) Betzig, E.; Finn, P. L.; Weiner, J. S. Appl. Phys. Lett. 1992, 60, 2484 the incident beam can be rotated by means of a $\lambda / 4$ plate and a polarizer. A small fraction of the incident power, about $0.3 \mathrm{nW}$, is emitted from the aperture into the far field. This small light source is placed near the sample, which is scanned by a homebuilt scanner based on multimorph piezo translators.

While scanning, the distance between probe and sample is kept constant at about $3 \mathrm{~nm}$ by a shear force feedback. Hereto the fiber is oscillating laterally with an amplitude of about 30 $\mathrm{nm}$ at its resonance frequency (typically $10 \mathrm{kHz}$ ). Forces between probe and sample cause a decrease in amplitude and a phase shift of the oscillation when the tip approaches the surface to a few nanometers. Keeping the amplitude at a constant value results in a constant distance between probe and sample. Thus the shear force microscope provides an image of the topography with a high resolution which appears to be about $50 \mathrm{~nm}$ laterally and about $0.5 \mathrm{~nm}$ vertically in practice. The instrument is described in more detail elsewhere. ${ }^{28}$

This system is placed on a Zeiss Axiovert 135 TV inverted microscope. The fiber probe is used for the local excitation of the polymeric film with polarized light. The fluorescence from the film, which is in the order of $0.1 \mathrm{pW}$, is collected by an objective $(40 \times, 0.60$ numerical aperture) and detected with a photomultiplier tube (Hamamatsu R1463-01). A 200- $\mu \mathrm{m}$ pinhole in the image plane reduces the background signal. A dichroic filter set in the optical path blocks the laser light and transmits the fluorescence.

Because the linear polarization is not necessarily maintained in the multimode fiber, a revolving analyzer is placed in the optical path to measure the degree of polarization, $P=\left(I_{\max }-\right.$ $\left.I_{\min }\right) /\left(I_{\max }+I_{\min }\right)$, of the light emitted from the aperture before the fluorescence measurements are started. For practical reasons the analyzer is kept in the setup at the angle of maximal intensity during the measurements.

Both the height of the object and the local fluorescence are measured simultaneously while the sample is raster-scanned. The data are stored in a personal computer, which also generates the scan pattern.

\section{Results and Discussion}

In Figure 2 the topography and a simultaneously recorded fluorescence SNOM image of a DPDA film on a glass substrate are shown. Several polymer domains, with a thickness of a few nanometer, overlap in the $4.4 \mu \mathrm{m} \times$ $4.4 \mu \mathrm{m}$ scan, as seen in the shear force image, Figure 2a. A large domain of polymer film is observed at the left with cracks in a specific direction. These cracks may be formed by mechanical distortion of the film during the transfer to the substrate. ${ }^{18}$ A closer examination of the surface of this polymer domain reveals a striped pattern oriented

(28) Moers, M. H. P.; Ruiter, A. G. T.; Van Hulst, N. F.; Bölger, B. Ultramicroscopy 1994, in press. 

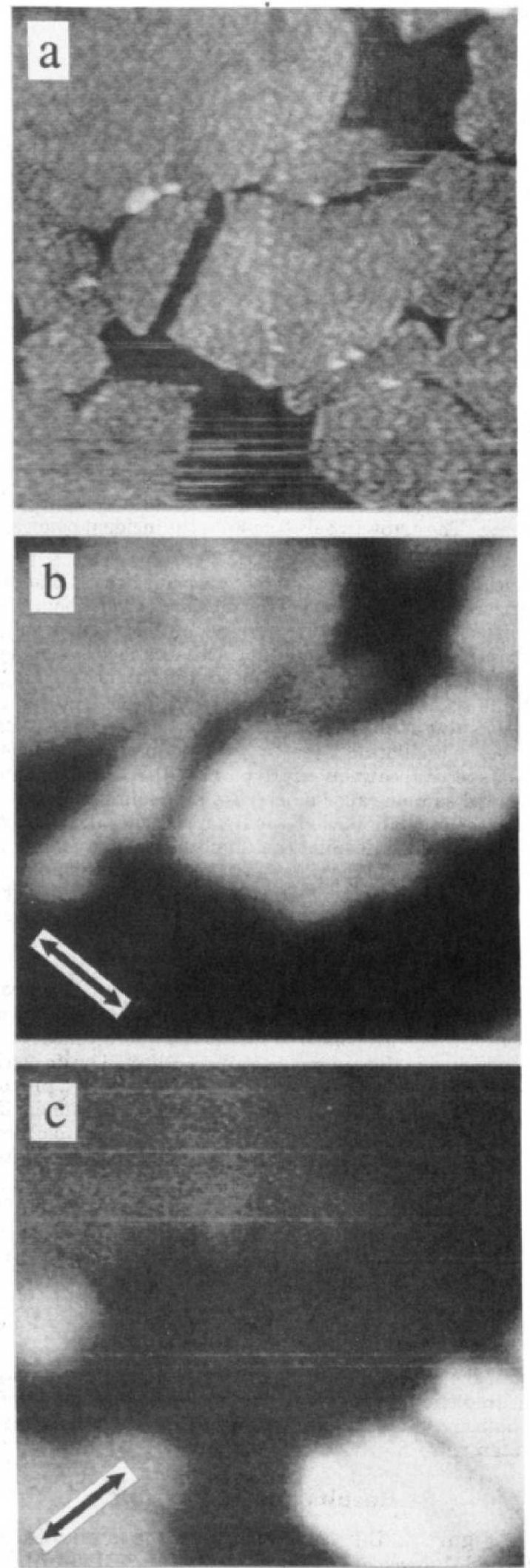

Figure 3. A $4 \mu \mathrm{m} \times 4 \mu \mathrm{m}$ scan of the same LB film of DPDA: the shear force image (a) and two near-field fluorescence images (b, c) with different incident polarizations are shown. The polarization directions are denoted by arrows. The relative intensities of the several domains change upon rotation of the polarization because of the high anisotropy of the polydiacetylene film.

parallel to the cracks. This has been reported by other groups $^{17-19}$ and probably is not pure topography. Local variations of lateral forces might play a role, as suggested

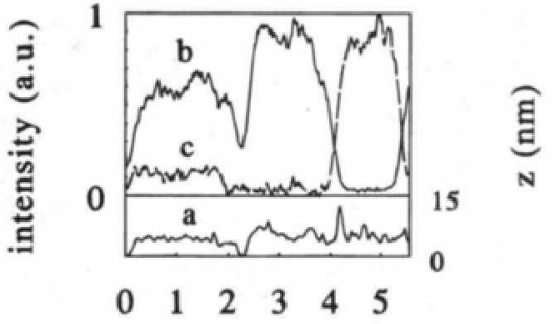

$\mathrm{x}$ (micron)

Figure 4. Fluorescence intensities of parts b and $c$ of Figure 3 and the height profile of Figure $3 \mathrm{a}$ along the diagonal (from upper left to lower right) are shown. The anisotropy of the polymeric film is clearly demonstrated by the fluorescence intensity upon rotation of the incident polarization.

previously. ${ }^{18,19}$ Adjacent to this large domain several other fragments of polymer are observed in the right part of the image.

The near-field fluorescence image, Figure $2 \mathrm{~b}$, has been obtained with 488-nm excitation and a polarization $(P=$ $0.6)$ roughly in the direction of the cracks, as denoted by the arrow. A 450-490 $\mathrm{nm}$ dichroic mirror in combination with a 520-nm long-pass filter in the optical path selects the emitted light. The fluorescence of the large domain on the left is clearly visible with a fairly high resolution of about $100 \mathrm{~nm}$, whereas the other, smaller, fractions do not fluoresce at all. The fluorescence intensity in this image is $\sim 1 \mathrm{fW}$. Rotation of the polarization causes a decrease in intensity of the large domain, while the smaller fractions remain dark. It is well possible that these smaller fractions consist of monomeric lipid. It is known that only a fraction of the lipid is polymeric and that only a fraction of the polymer is fluorescent. ${ }^{2,7}$

Comparison of the topography and the fluorescence micrograph in Figure 2 obviously reveals complementary information about the sample. In the force image, domains are observed which do not fluoresce, as appears from the optical image. The combination of both techniques allows a discrimination between the different phases and their orientations in the subwavelength scale.

Figure 3 shows another scan of the same sample, $4 \mu \mathrm{m}$ $\times 4 \mu \mathrm{m}$ in size. In the shear force image, Figure $3 \mathrm{a}$, several domains of DPDA are visible. Parts b and $c$ of Figure 3 give fluorescence micrographs of the same area with mutually perpendicular directions of incident linear polarization $(P=1)$, as denoted by the arrows in the images. Excitation is with $514 \mathrm{~nm}$, and a $510-560 \mathrm{~nm}$ dichroic mirror with 590-nm long-pass filter is placed in the optical path.

The high anisotropy of polymerized diacetylenic films is clearly demonstrated in these measurements. Absorption and emission dipole moments of polydiacetylenes are oriented parallel to the polymer backbone. Due to the crystallinity and the polymer packing of the LangmuirBlodgett film, the polymer backbones in a distinct domain have a uniform distribution, parallel to the plane. ${ }^{1,4}$ Upon rotation of the incident polarization, the fluorescence intensities change as $\cos ^{2}(\vartheta) \cos ^{2}(\varphi), \vartheta$ being the angle between incident polarization and absorption moment and $\varphi$ the angle between emission moment and analyzer. Because the absorption and emission moments both are oriented along the polymer backbone, and the analyzer is set parallel to the incident polarization, this equation simplifies to $\cos ^{4}(\vartheta)$.

This is depicted more quantitatively in Figure 4 . The fluorescence intensities along the diagonal of parts $b$ and c of Figure 3 are shown together with the line profile in 


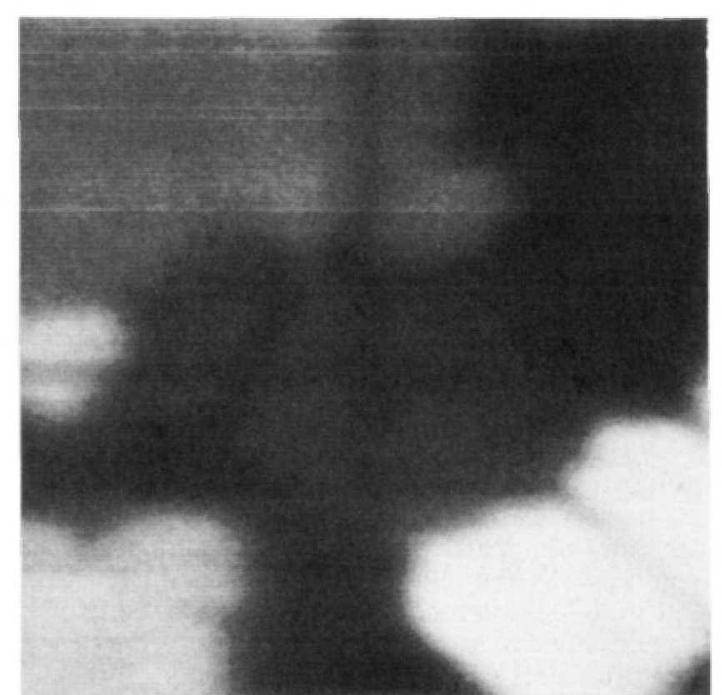

Figure 5. A $4 \mu \mathrm{m} \times 4 \mu \mathrm{m}$ fluorescence micrograph of the same area as Figure 3. A reduced fluorescence intensity is observed as a dark "+" along the central axes of the scan. This is due to photobleaching during the several line scans in both directions that are necessary to adjust the instrument.

the force image. The fluorescence intensity of each domain varies with the polarization.

When the fluorescence micrographs are compared with the corresponding force image, it is revealed that domains which appear to be one in the force image actually are formed by more domains that are attached to each other. Optical properties of the lipid film can be related to the topological structure.

Finally, in Figure 5 a reduced fluorescence intensity is observed as a dark "+" along the central axes of the image.
In this particular scan, of the same area as Figure 3, the intensity emitted from the aperture has been increased to about $1 \mathrm{nW}$. The decrease in fluorescence intensity along the central axes of Figure 5 is due to photobleaching during the several line-scans in both directions that are necessary to adjust the instrument. This capability to write and read structures in a polymer film offers the prospect of high-resolution photolithography and highdensity data storage. ${ }^{29}$

\section{Conclusion}

In this paper we have shown the application of a scanning near-field optical microscope in the study of the optical properties of thin lipid films. Fluorescence micrographs have been presented which show optical contrast with a resolution exceeding classical far-field optics. In particular the combination with a force microscope is powerful, because correlative imaging is possible. Comparison of the fluorescence micrographs with the force images yields further information on the sample. The complementary information afforded by this combination is valuable for Langmuir-Blodgett research and development particularly in view of potential applications.

Acknowledgment. The authors thank Frans Segerink, Kees van der Werf, and Eric Schipper for their help with the construction of hard- and software. The samples have been prepared by Uli Hoffmann of the Technical University of Munich. This project is mainly supported by the Dutch Foundation for Fundamental Research (FOM). Dr. Gaub is supported by the Deutsche Forschungsgemeinschaft.

(29) Betzig, E.; Trautman, J. K.; Wolfe, R.; Gyorgy, E. M.; Finn, P. L. Appl. Phys. Lett. 1992, 61, 142. 